\title{
Chimeric DCL1-Partnering Proteins Provide Insights into the MicroRNA Pathway
}

\author{
Rodrigo S. Reis ${ }^{1,2 *}$, Andrew L. Eamens ${ }^{3}$, Thomas H. Roberts ${ }^{2}$ and \\ Peter M. Waterhouse ${ }^{1,4 *}$
}

${ }^{1}$ School of Biological Sciences, University of Sydney, Sydney, NSW, Australia, ${ }^{2}$ Department of Plant and Food Sciences, Faculty of Agriculture and Environment, University of Sydney, Sydney, NSW, Australia, ${ }^{3}$ School of Environmental and Life Sciences, University of Newcastle, Callaghan, NSW, Australia, ${ }^{4}$ Centre for Tropical Crops and Biocommodities, Queensland University of Technology, Brisbane, QLD, Australia

OPEN ACCESS

Edited by:

Richard A. Jorgensen,

University of Arizona, USA

Reviewed by:

lain Robert Searle,

The University of Adelaide, Australia

Bernie Carroll,

The University of Queensland,

Australia

*Correspondence:

Rodrigo S. Reis

rodrigo.siqueirareis@unil.ch;

Peter M. Waterhouse

peter.waterhouse@qut.edu.au

Specialty section:

This article was submitted to Plant Genetics and Genomics,

a section of the journal

Frontiers in Plant Science

Received: 14 July 2015

Accepted: 14 December 2015

Published: 06 January 2016

Citation:

Reis RS, Eamens AL, Roberts TH and Waterhouse PM (2016) Chimeric

DCL1-Partnering Proteins Provide Insights into the MicroRNA Pathway.

Front. Plant Sci. 6:1201.

doi: 10.3389/fpls.2015.01201
In Arabidopsis thaliana, efficient microRNA (miRNA) production requires DICERLIKE1 (DCL1) with the assistance of a partnering protein, DOUBLE-STRANDED RNA BINDING1 (DRB1) or DRB2. The presence of either of these DRB proteins is crucial to determine the mode of action of a miRNA; i.e., cleavage or translation inhibition. Here we studied the structural determinants for the role of DRB1 and DRB2 in the miRNA pathway. We developed a series of chimeric vectors encoding different functional domains of DRB1 and DRB2, and expressed these in the drb1 mutant background in Arabidopsis under the control of the native DRB1 promoter. Complementation of the drb1 developmental phenotype was used to assess the biological role that each functional domain of DRB1 and DRB2 mediates in the miRNA-guided transcript cleavage pathway. The DRB1 amino acid sequence differs considerably to that of DRB2, and analysis of drb1 transgenic lines revealed that the first dsRNA-binding domains of DRB1 and DRB2 are functionally similar; in contrast, the dsRBD2 of DRB1 and DRB2 appear functionally distinct. Our bioinformatic analysis further suggests that the C-terminal domain of DRB2 mediates a functional role in the miRNA pathway, whereas its counterpart in DRB1 is known to be dispensable. Our results provide evidence for the differences between DRB1 and DRB2 proteins in vivo, which may be essential for the selection of the miRNA regulatory mechanisms, and suggest that these features are conserved among land plants.

Keywords: DRB1, HYL1, DRB2, miRNA, dsRNA binding domain, chimera

\section{INTRODUCTION}

DOUBLE-STRANDED RNA BINDING1 (DRB1) is a well characterized partnering protein of DCL1 and is required for accurate and efficient processing of miRNA/miRNA* duplexes from their respective precursor transcripts (Kurihara et al., 2006; Dong et al., 2008). DRB1 also mediates the preferential selection of miRNA guide strands over the corresponding duplex strand (the miRNA* passenger strand) for loading into the ARGONAUTE1 (AGO1)-catalyzed RNA-induced silencing complex (RISC; Eamens et al., 2009). DRB2 is also a DCL1 partnering protein (Eamens et al., 2012). We have recently shown that DRB1 is required only for miRNA-guided transcript cleavage, whereas DRB2 represses DRB1 transcription and is required for the translational inhibition 
pathway (Reis et al., 2015). Thus, although DCL1 is the ribonuclease III that excises miRNA/miRNA* duplexes from precursor transcripts, its DRB partnering protein defines the miRNA mode of action.

In Arabidopsis, genes encoding three additional DRB proteins-DRB3, DRB4, and DRB5-are present and each DRB is characterized by two amino-terminal dsRNA-binding domains (dsRBDs; Curtin et al., 2008). dsRBD-containing proteins have been identified in most eukaryotes, are typically 70 amino acid residues in length, fold into an $\alpha \beta \beta \beta \alpha$ structure, mediate dsRNA recognition and binding, and can also bridge protein-protein interactions (Chang and Ramos, 2005). In a canonical dsRNA binding domain, three regions of contact with the dsRNA molecule can be distinguished: regions 1 and 2 bind the dsRNA minor groove, while region three binds the major groove (Gleghorn and Maquat, 2014). The regions are characterized by positive electrostatic surface, with region two featuring a $\mathrm{GPxH}$ motif.

The structures of both dsRBD domains of DRB1, termed dsRBD1 and dsRBD2, respectively, have been determined (Yang et al., 2010). These revealed that dsRBD1 adopts a canonical dsRBD structure; i.e., it has the structural features found in most dsRNA binding domains. Interestingly, dsRBD2 is structurally distinct and its predicted low affinity for the dsRNA substrates of DRB1 has been explained by deviations from the canonical structure in region 2 (the loop that recognizes the dsRNA minor groove), and to a lesser extent in the $\alpha$-helices that recognize both the minor and major dsRNA grooves (Yang et al., 2010). The same authors also showed that DRB1 binds 21nucleotide (nt) dsRNA as a homodimer, probably mediated by its dsRBD2. More recently, DRB1 homodimerization has been further characterized, with the role of dsRBD2 shown to be crucial to ensure the position of DCL1-catalyzed pri-miRNA cleavage (Yang et al., 2014). Surprisingly, disruption of DRB1 homodimerization did not impair DRB1 interaction with DCL1, or the pri-miRNA binding affinity of DRB1.

Engineered chimeric genes coding for different combinations of dsRBDs have been widely used to study their functions in humans and plants. In humans, a single dicer protein processes both pre-miRNAs and pre-siRNAs in association with DRB proteins, namely protein activator of PKR (PACT) (Lee et al., 2006) and trans-activation response RNA-binding protein (TRBP; Chendrimada et al., 2005; Haase et al., 2005), respectively. Animal miRNAs primarily guide translation inhibition, while short interfering RNAs (siRNAs) guide transcript cleavage. Lee et al. (2013) showed that in humans this dichotomy is partially aided by Dicer association with either PACT or TRBP. PACT was found to inhibit the processing of pre-siRNA substrates by Dicer, and experiments with chimeric versions of PACT and TRBP demonstrated that the two N-terminal RNA-binding domains of each protein confer their differential function. Chimeric versions of DRB proteins have also been used to unravel the structural components of Caenorhabditis elegans RDE-4 required to bind dsRNA, interact with dicer DCR-1 and activate DCR-1 (Parker et al., 2008). Interestingly, a chimeric DRB protein containing the canonical TRBP2 dsRBD2 fused to the non-canonical DRB1 dsRBD2 compensated for DRB1 dsRBD1 by rescuing the $d r b 1$ severe phenotype (Yang et al., 2010), providing evidence that certain dsRNA binding domains are primarily involved in the recognition and binding of dsRNA; e.g., DRB1 dsRBD1.

In this study, we demonstrate that the dsRBD1 domain of DRB1 is functionally similar to the corresponding domain in DRB2, whereas their dsRBD2 domains are functionally distinct. We also show that, while the C-terminal of DRB1 appears functionally redundant, its counterpart in DRB2 is functional. These results provide insights into the structural determinants of DRB1 and DRB2 activity in vivo, and into the miRNA-guided transcript cleavage pathway.

\section{MATERIALS AND METHODS}

\section{Plant Lines and Growth Conditions}

The drb1 T-DNA knockout insertion has been described previously (Curtin et al., 2008; Eamens et al., 2012). Plant lines were cultivated under standard growth conditions of $16 \mathrm{~h}$ light $/ 8 \mathrm{~h}$ dark at a constant temperature of $24^{\circ} \mathrm{C}$. Prior to soil transfer, all Arabidopsis lines were germinated on Murashige and Skoog (MS) agar media containing 1\% sucrose for PCR-based genotyping to confirm genetic background. DNA oligonucleotides used as primers for PCR-based genotyping are listed in Supplementary Material.

\section{Germination Under Abscisic Acid (ABA) Treatment}

Arabidopsis Col-0 wild-type, mutant and transgenic line seeds were placed on filter paper saturated with either water (control) or $0.5 \mu \mathrm{M}$ abscisic acid (ABA), incubated at $4^{\circ} \mathrm{C}$ for $48 \mathrm{~h}$, and then transferred to growth cabinets for germination under standard growth conditions.

\section{Protein Sequence Alignment}

Putative Arabidopsis thaliana DRB1 and DRB2 ortholog protein sequences were obtained using the Phytozome database (Goodstein et al., 2012) and the basic local alignment search tool (BLAST; Altschul et al., 1990). Sequence alignments were performed using the default parameters of Clustal W (Larkin et al., 2007). The evolutionary tree time scale was based on a previous report (Clarke et al., 2011). The complete list of ortholog proteins is presented in Supplementary Material.

\section{Prediction of dsRNA Binding Domain Structure}

Secondary structure predictions for dsRNA binding domains of DRB2, DRB3, DRB4 and DRB5 were performed using the default parameters of the I-TASSER online server (Roy et al., 2010).

\section{Construction of Expression Vectors and Plant Transformation}

The construction of the expression vectors used to transform drb1 mutants was performed using standard cloning techniques using Gateway ${ }^{\circledast}$ cloning (Invitrogen) and synthesized DNA sequences. The binary vector used to transform the plants 


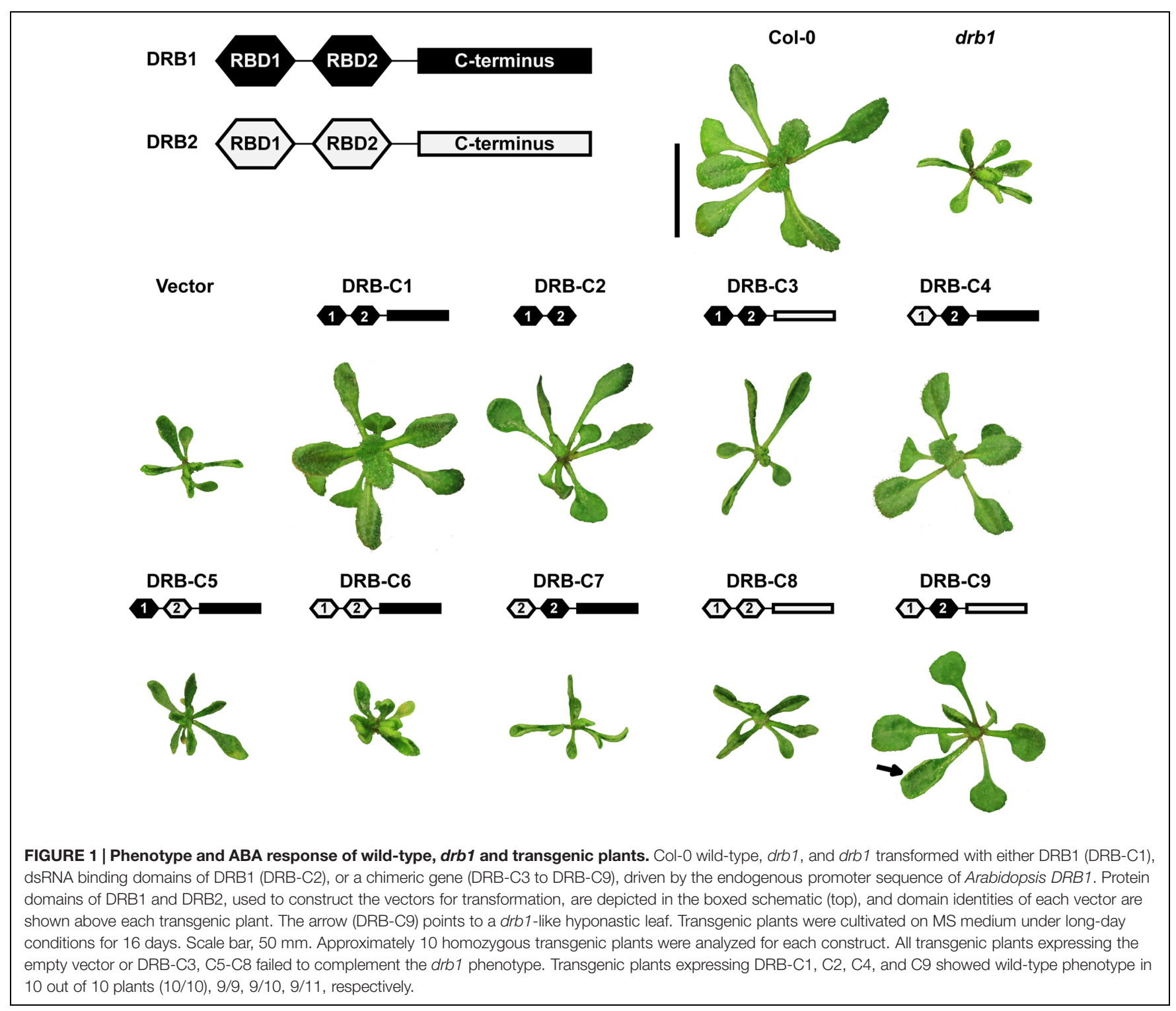

was a Gateway vector, pKCTAP, obtained from Plant Systems Biology (VIB, Belgium; http://gateway.psb.ugent.be/), which has been previously described (Van Leene et al., 2007). The selectable marker cassette from pORE-O1 (Coutu et al., 2007), containing a Pat gene driven by $P_{H P L}$ (A. thaliana hydroperoxide lyase promoter), was amplified using oligos that contained overhanging restriction sites for RsrII at both $5^{\prime}$ and $3^{\prime}$ ends. pKCTAP was digested with RsrII and the Pat gene cassette was sticky-end ligated.

To prepare the gene constructs (chimeras) to be inserted into the modified pKCTAP, a series of $\sim 500$ nt DNA sequences, termed gBlocks ${ }^{\circledR}$, were designed in-house and synthesized by Integrated DNA Technologies (IDT). Each gBlock contained sequences coding the dsRNA binding domain of DRB1 and/or DRB2 as listed and described in Supplementary Material. The gBlocks were designed to contain 5 HindIII and MfeI and a 3 NheI restriction site, which were subjected to restriction enzymatic digestion, followed by ligation, to aid their insertion into a vector containing either the DRB1 (MfeI and NheI) or DRB2 (HindIII and NheI) sequence. The obtained constructs, as well as the dsRNA binding domains of $D R B 1, D R B 1$ full-length, and $D R B 2$ full-length sequences, were amplified using a pair of primers designed to introduce a $5^{\prime}$ CACC overhanging sequence to allow their directional cloning into $\mathrm{pENTR/D-TOPO}{ }^{\circledR}$ (Invitrogen).

The $D R B 1$ promoter region, containing the $5^{\prime} \mathrm{UTR}$ of $D R B 1$ and $538 \mathrm{nt}$ genomic sequence, as previously described (Curtin et al., 2008), was modified to also include the first exon and intron of the $D R B 1$ gene. The longer $D R B 1$ promoter region was amplified from genomic DNA using oligos that added $5^{\prime}$ SphI and 3' SalI overhanging restriction sites. The PCR-amplified sequence was digested and ligated into a modified pEN::L4-2-R1 (also obtained from Plant Systems Biology, VIB). The pEN::L4-2$\mathrm{R} 1$ vector originally encodes the cauliflower mosaic virus (CaMV) $35 \mathrm{~S}$ promoter; thus, to remove the $35 \mathrm{~S}$ promoter, $\mathrm{pEN}: \mathrm{L} 44-2-\mathrm{R} 1$ 

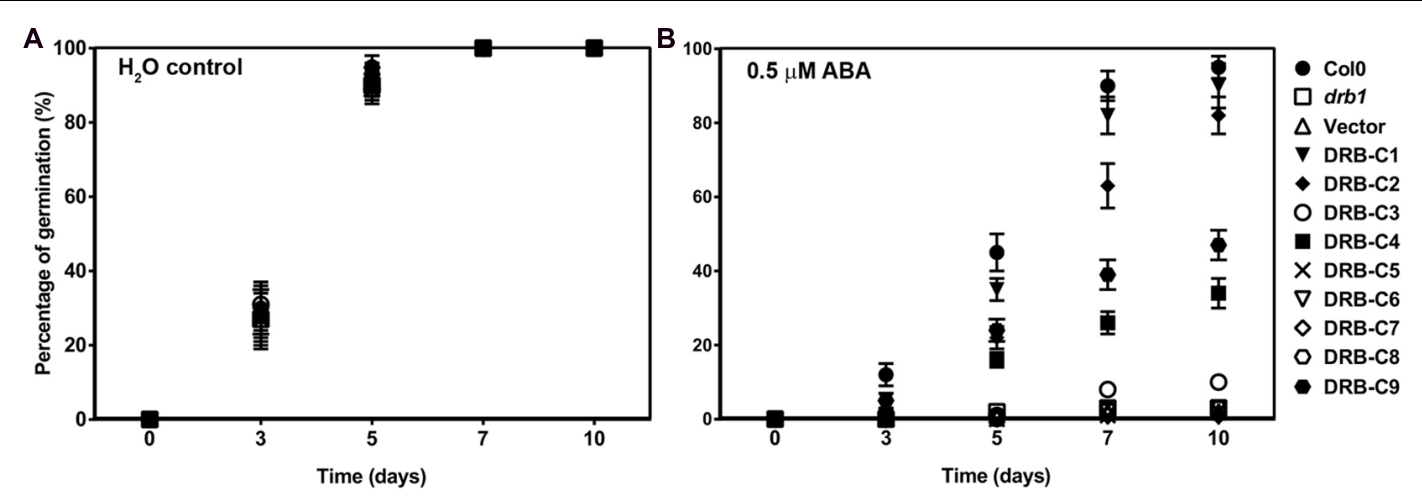

FIGURE 2 | Effects of exogenous ABA on wild-type, drb1 and transgenic plants. Seeds of wild-type, drb1 mutant and drb1 transgenic lines were germinated on filter paper saturated with either water $(\mathbf{A})$ or $0.5 \mu \mathrm{M} \mathrm{ABA}(\mathbf{B})$. Seeds were incubated at $4^{\circ} \mathrm{C}$ for $48 \mathrm{~h}$ and then transferred to room temperature for assessment of their germination efficiency over a period of 10 days $(n>100, \pm$ SD).

was amplified using oligos specific to its L4 and R1 att site pairs oriented to amplify the entire vector except the $35 \mathrm{~S}$ promoter.

The modified linear vector was digested with SpeI (restriction site included via PCR) and re-circularized, resulting in an L4/R1containing vector that contained a multiple cloning site (MCS), which was also included via PCR. This vector was then digested with SphI and SalI and ligated with the longer DRB1 promoter sequence, generating the pEN::L4-DRB1pro-R1. Finally, gateway cloning was performed using the entry vectors $\mathrm{pENTR/D-TOPO}$ containing a chimeric gene, pEN::L4-DRB1pro-R1, pEN::R2GStag-L3, and the modified destination vector, pKCTAP. The resulting expression vector was used to transform $d r b 1$ mutant plants via Agrobacterium-mediated transformation using the floral dip method (Clough and Bent, 1998). Plants were selected for resistance to the herbicide glufosinate. The sequences of oligos used are listed in Supplementary Material.

\section{RNA Isolation}

For all RNA analyses, total RNA was isolated using TRIzol Reagent (Invitrogen) according to the manufacturer's instructions.

\section{Real Time RT-PCR}

Synthesis of cDNA for real-time reverse-transcription PCR (RT-PCR) was performed using SuperScript ${ }^{\circledR}$ III Reverse Transcriptase (Life Technologies) following the manufacturer's instructions. RT-PCR was performed using Brilliant III SYBR ${ }^{\circledR}$ MM according to the Agilent Technologies protocol. The sequences of oligos are listed in Supplementary Material.

\section{miRNA Real Time RT-PCR}

RT-PCR for the quantification of miRNA accumulation was performed according to a previous report (Chen et al., 2005). SuperScript ${ }^{\circledR}$ III Reverse Transcriptase (Life Technologies) and Brilliant III SYBR ${ }^{\circledR}$ MM (Agilent Technologies) were used to perform the cDNA synthesis and RT-PCR, respectively, following the manufacturers' instructions. Arabidopsis SnoR101 was used to normalize the miRNA accumulation. The sequences of primers are listed in Supplementary Material.

\section{RESULTS AND DISCUSSION}

\section{Expression of Chimeric Gene Series in drb1 Plants}

Arabidopsis plants defective in the activity of DRB1-drb1 knockout mutant plants-exhibit pleiotropic developmental defects characterized by reduced overall size and hyponastic rosette leaves (Lu and Fedoroff, 2000; Wu et al., 2007). To investigate the structural components that direct the functional activity of DRB1, and that differentiate it from DRB2 in the Arabidopsis miRNA pathway, we transformed $d r b 1$ plants with a series of chimeric vectors. Each vector harbored coding sequences for DRB1 and DRB2 as full-length, truncations or rearrangements. The in vivo expression of these chimeric vectors, termed DRB-C1 to DRB-C9, was driven by the native DRB1 promoter. Homozygous plants were identified in the T2 generation and further selected by gene expression of chimeras relative to endogenous DRB1 in wild-type Arabidopsis (Col$0)$. The assessment of each chimera's expression level was performed such that all selected transgenic plants had chimeric genes at levels similar to endogenous DRB1 in wild-type plants. Transgenic plants were then visually assessed for comparison to wild-type and $d r b 1$ plants.

Previous work has shown that in vivo expression of both $\mathrm{N}$-terminal dsRBDs of DRB1 lacking its C-terminal region, when driven by the constitutive CaMV 35S promoter, was sufficient for complementation of the severe $d r b 1$ developmental phenotype ( $\mathrm{Wu}$ et al., 2007). Here, we observed that the expression of DRB1 full-length (DRB-C1) or DRB1 N-terminal dsRBDs (DRB-C2), driven by the $D R B 1$ endogenous promoter in a $d r b 1$ mutant background ( $d r b 1 / \mathrm{DRB}-\mathrm{C} 1$ and $d r b 1 / \mathrm{DRB}-$ C2 plants), also allowed for phenotypic complementation of this mutant (Figure 1). This further confirms that the N-terminal dsRBDs fulfill the function of the whole DRB1. 


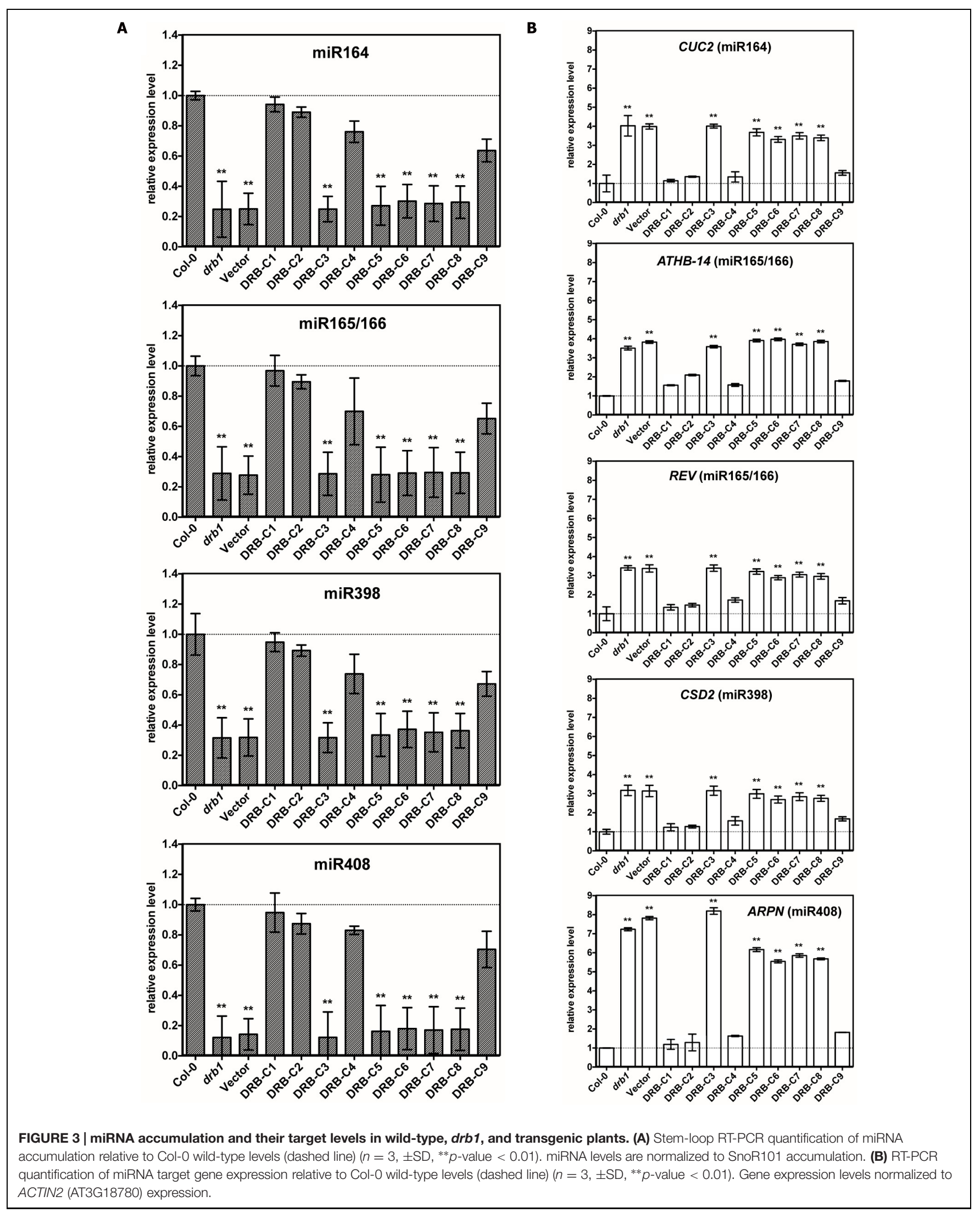




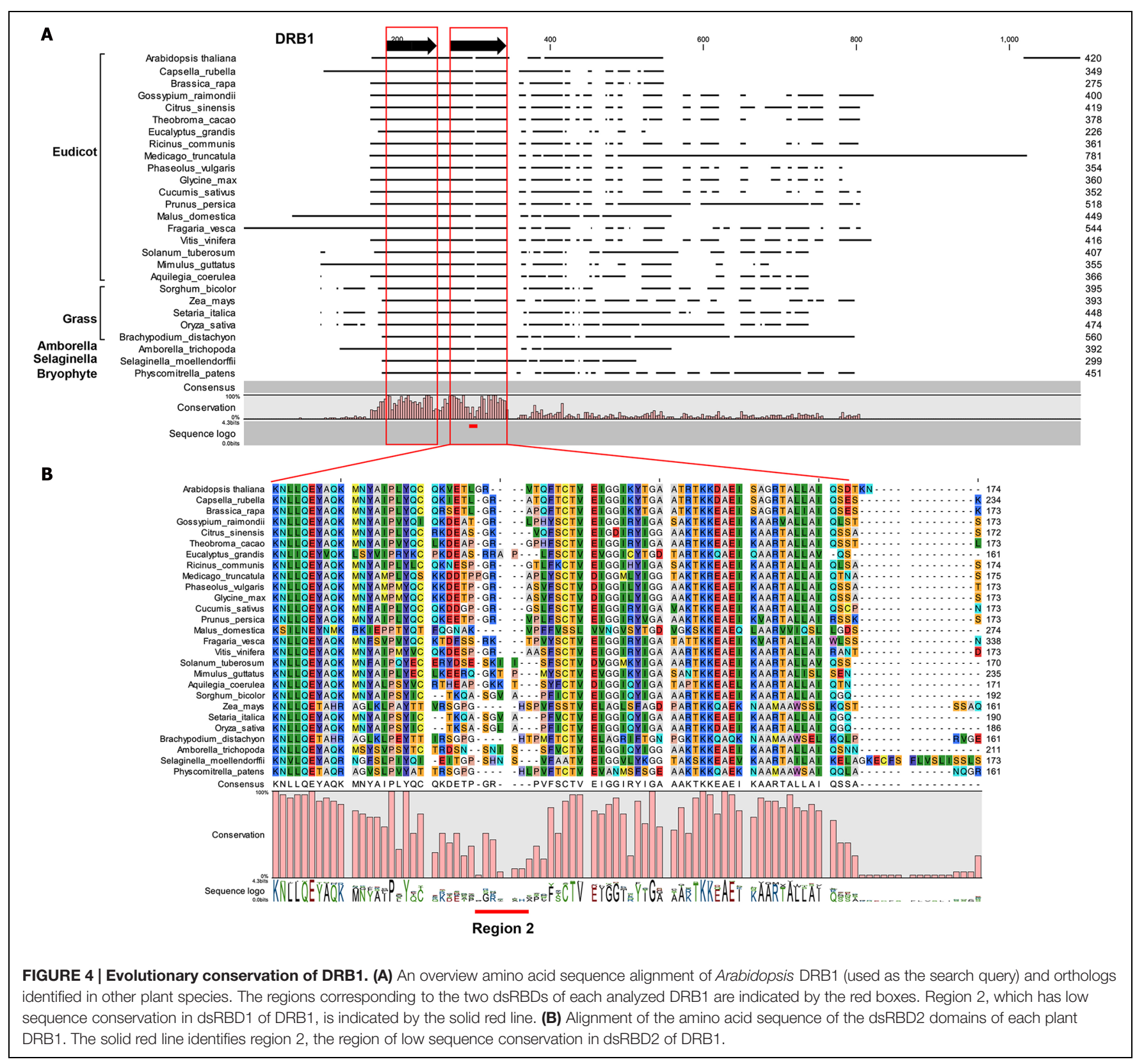

However, transformation of $d r b 1$ mutant plants with chimeric genes lacking the non-canonical DRB1 dsRBD2 (DRB-C5, C6 and C8) failed to complement the severe phenotype. Transgenic lines $d r b 1 / \mathrm{DRB}-\mathrm{C} 5, d r b 1 / \mathrm{DRB}-\mathrm{C} 6$ and $d r b 1 / \mathrm{DRB}-$ C8 displayed the $d r b 1$-like phenotype, showing that the noncanonical domain of DRB1 is essential for its in vivo activity. This also revealed that $D R B 2$ under control of the endogenous promoter of DRB1 (drb1/DRB-C8 plants) is not capable of complementing drb1 phenotype, whereas under control of the strong and constitutive $35 \mathrm{~S}$ promoter, $D R B 2$ can compensate for DRB1 null mutation (Eamens et al., 2012). This is in accordance with the lower affinity of DRB2 for DCL1 as compared with that of DRB1 protein (Kurihara et al., 2006), and further suggests that DRB2 can only compensate for
DRB1 at elevated levels in order to compensate for its lower affinity.

The DRB-C7 chimeric gene also failed to complement the $d r b 1$ phenotype. In the DRB-C7 chimeric gene, the canonical first dsRBD of DRB1 was replaced with DRB2 dsRBD2, which was predicted to fold into a canonical RBD (Figure 1). drb1/DRBC7 plants showed a drb1-like phenotype, suggesting that (i) DRB2 RBD2 is a non-canonical dsRNA binding domain or (ii) it mediates specific protein-protein interaction(s) different to those of DRB1. DRB1 interaction with its partnering proteins has been shown to require its second dsRNA binding domain (Yang et al., 2010); hence, it is likely that the second dsRNA binding domain of DRB2 plays a similar role in mediating protein-protein interactions. 
Although the C-terminal region of DRB1 appears to be dispensable for its function (Wu et al., 2007), the transformation of $d r b 1$ with chimeric genes harboring the DRB2 C-terminal region (DRB-C3 and DRB-C9) resulted in different phenotypes. DRB-C3 has the DRB1 dsRNA binding domains fused to the C-terminus of DRB2, and $d r b 1 / \mathrm{DRB}-\mathrm{C} 3$ plants displayed a $d r b 1$ like phenotype (Figure 1). The DRB-C9 chimeric gene, however, is similar to DRB-C3, with the difference that it has the first dsRBD of DRB1 replaced by DRB2 dsRBD1. Interestingly, the $d r b 1 / \mathrm{DRB}-\mathrm{C} 9$ phenotype was closely related to wild-type, but also had some hyponastic leaves, characteristic of $d r b 1$ mutants. These results show that the DRB2 C-terminus can impair DRB1 function in the absence of DRB2 dsRBD1, suggesting that these domains may interact. In addition, the DRB-C4 chimeric gene has the first $\mathrm{RBD}$ of $\mathrm{DRB} 1$ replaced by $\mathrm{DRB} 2$ $\mathrm{RBD} 1$, and $d r b 1 / \mathrm{DRB}-\mathrm{C} 4$ is wild-type in appearance. Although it is possible that certain chimeric combinations may lead to disruption of protein tertiary structure, this is unlikely as several dsRBD chimeric combinations have been previously shown to be functional (Parker et al., 2008; Yang et al., 2010; Lee et al., 2013). However, it is still possible that the chimeric genes that failed to complement the $d r b 1$ phenotype are non-functional because of improper folding, altered posttranscriptional modification (e.g., phosphorylation) or posttranscriptional regulation (e.g., loss of binding site). These are all interesting avenues for further studies, and our results present a comprehensive picture of domain swapping that, due to incompatibility or non-functionality, give combinations impaired in their ability to complement the $d r b 1$ phenotype. These results also suggest that the dsRBD1 of DRB1 and DRB2 are functionally similar in the miRNA pathway.

\section{Abscisic Acid Treatment of the drb1 Transgenic Lines}

It has been previously demonstrated that $d r b 1$ seed germination is inhibited by exogenous ABA application (Lu and Fedoroff, 2000). To further characterize the transgenic populations resulting from the in vivo expression of the chimeric vector series, T3 seed was collected from homozygous T2 transgenic lines and the germination efficiency of each seed pool assessed via germination on filter paper soaked with either water or $0.5 \mu \mathrm{M}$ ABA (Figure 2). In contrast to seeds germinated on water-soaked filter paper, on which all assessed $d r b 1$ transgenic lines germinated efficiently, all plant lines showed reduced or completely abolished germination in the presence of ABA (Figure 2). In accordance with the phenotypic analysis (Figure 1), transgenic lines drb1/DRB-C1, drb1/DRB-C2, drb1/DRB-C4 and drb1/DRBC9, all of which displayed wild-type-like phenotypes, showed limited sensitivity to ABA, as did Col-0 plants (Figure 2). However, seeds collected from transgenic lines that displayed $d r b 1$-like phenotypes, including $d r b 1 / \mathrm{DRB}-\mathrm{C} 3, d r b 1 / \mathrm{DRB}-\mathrm{C} 5$, $d r b 1 / \mathrm{DRB}-\mathrm{C} 6, d r b 1 / \mathrm{DRB}-\mathrm{C} 7$, and $d r b 1 / \mathrm{DRB}-\mathrm{C} 8$ plants, were highly sensitive to the ABA treatment (Figure 2). Furthermore, the ABA-sensitivity displayed by these transgenic lines was highly similar to the ABA hypersensitivity of non-transgenic drb1 plants (Figure 2). Taken together, these results reveal a clear correlation between complementation of the $d r b 1$ phenotype and ABA sensitivity; i.e., $d r b 1$ transgenic lines that complemented the drb1 phenotype were not hypersensitive to $A B A$, whereas the transgenic lines that failed to complement the $d r b 1$ phenotype were hypersensitive.

\section{miRNA Accumulation and Target Gene Expression in Plants Transformed with a Chimeric Vector Series}

At the molecular level, drb1 mutants are characterized by reduced miRNA accumulation and de-repression of miRNA target gene expression (Han et al., 2004; Kurihara et al., 2006). The accumulation of four highly conserved and wellcharacterized plant miRNAs, and the expression of their target genes, was therefore assessed in $d r b 1$ transgenic lines expressing the chimeric vector series. As observed for the ABA sensitivity assay, levels of miRNA accumulation and miRNA target gene expression were strongly correlated with the phenotype displayed by each of the $d r b 1$ transgenic lines (Figure 3 ). In the transgenic lines $d r b 1 / \mathrm{DRB}-\mathrm{C} 1, d r b 1 / \mathrm{DRB}-\mathrm{C} 2, d r b 1 / \mathrm{DRB}-\mathrm{C} 4$, and $d r b 1 / \mathrm{DRB}-\mathrm{C} 9$, which displayed wild-type-like phenotypes, accumulation of miR164, miR165/166, miR398 and miR408 and target gene expression of CUP SHAPED COTLEDONS2 (CUC2; miR164), ARABIDOPSIS THALIANA HOMEOBOX PROTEIN14 (ATHB-14; miR165/166), REVOLUTA (REV; miR165/166), COPPER/ZINC SUPEROXIDE DISMUTASE2 (CSD2; miR398), and PLANTACYANIN (ARPN; miR408) were at approximately wild-type levels (Figures 3A,B). Furthermore, the degree of drb1 phenotype complementation displayed by $d r b 1 / \mathrm{DRB}-\mathrm{C} 1$, $d r b 1 / \mathrm{DRB}-\mathrm{C} 2, d r b 1 / \mathrm{DRB}-\mathrm{C} 4$ and $d r b 1 / \mathrm{DRB}-\mathrm{C} 9$ transgenic lines (Figure 1) was supported by the molecular analyses presented in Figures 3A,B. $d r b 1 / \mathrm{DRB}-\mathrm{C} 1$ transgenic lines that expressed the full-length DRB1 transgene and displayed the highest degree of complementation (Figure 1) gave miRNA accumulation and target gene expression levels equivalent to those determined for wild-type plants. $d r b 1 / \mathrm{DRB}-\mathrm{C} 9$ transgenic lines, which expressed the DRB-C9 chimeric vector that housed only the second dsRBD of DRB1, and developed rosette leaves with mild hyponasty, had only slightly reduced miRNA accumulation and a corresponding mild elevation in miRNA target gene expression. Furthermore, $d r b 1$ transgenic lines that expressed chimeric vectors DRB-C3, DRB-5, DRB-C6, DRB-C7 or DRB-C8, and displayed drb1like phenotypes, gave miRNA and target gene expression levels equivalent to those observed in non-transgenic $d r b 1$ mutant plants. Taken together, the phenotypic and molecular analysis (Figures 1-3) showed that (i) DRB1 and DRB2 dsRBD1 are functionally similar, (ii) their dsRBD2s appear functionally distinct, and (iii) their C-terminal regions appear to perform different functions.

\section{Evolutionary Conservation of DRB1 and DRB2}

DOUBLE-STRANDED RNA BINDING1 and DRB2 play a major role in determining the silencing fate of a miRNA (Reis et al., 2015). Here we identified the structural components of DRB1 and DRB2 required for the miRNA-guided transcript cleavage pathway. To further characterize these proteins, we 


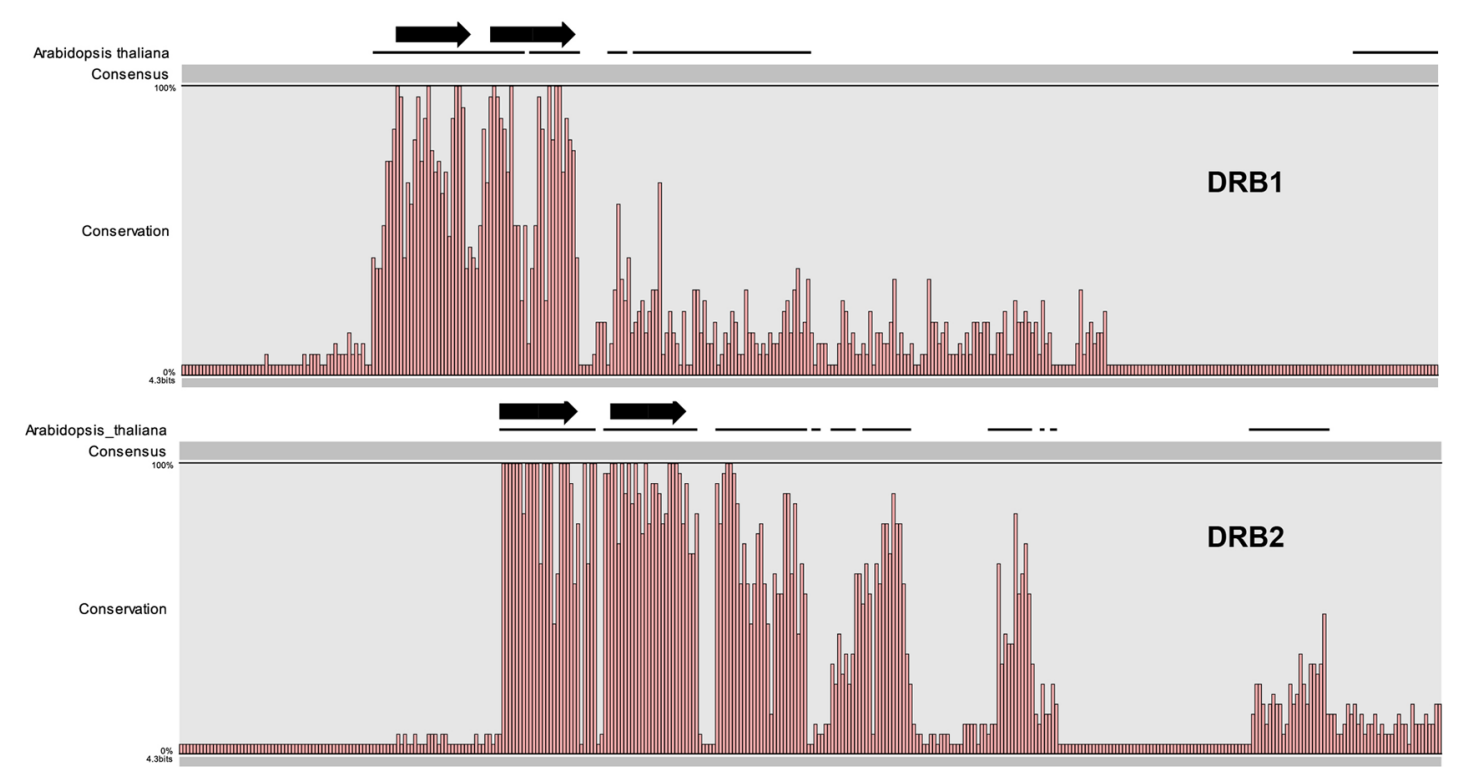

FIGURE 5 | Comparison of the conservation between identified DRB1 and DRB2 orthologs. The full-length sequences of DRB1 and DRB2 orthologs were scaled to the same size for direct comparison and the level of amino acid sequence conservation across these two DRB proteins schematically shown.

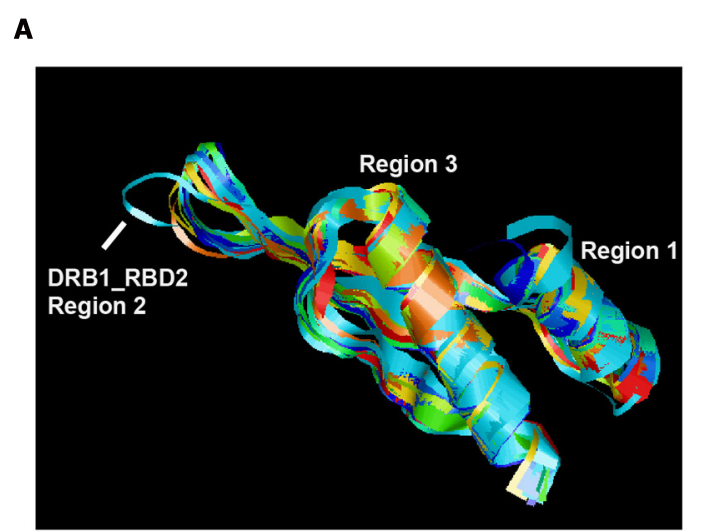

C
B

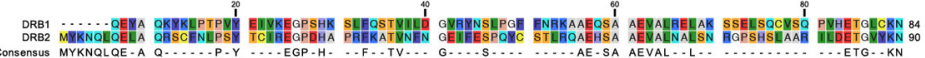

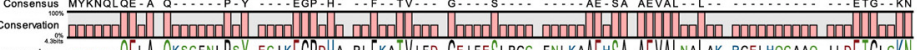
soevonoc oogo

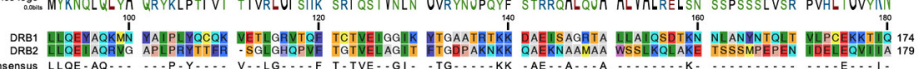

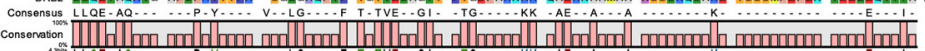

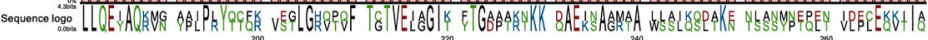

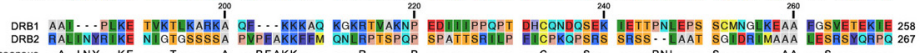

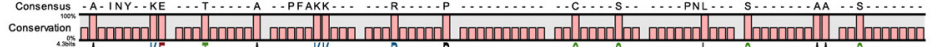

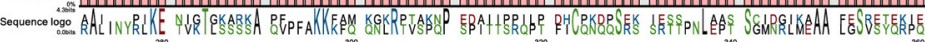

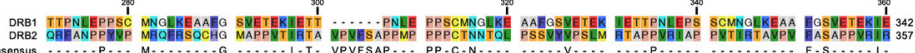
Consessus

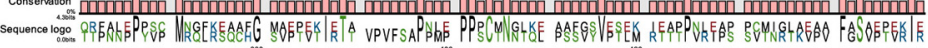

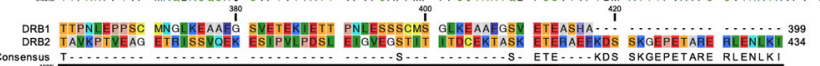

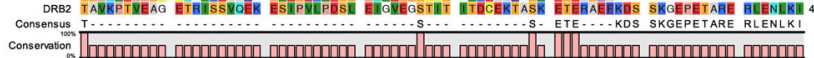
conseraton

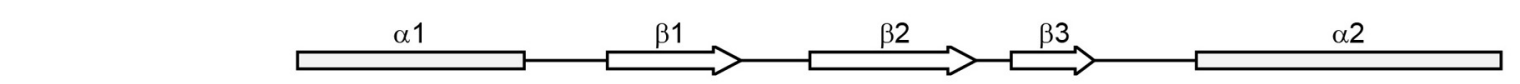

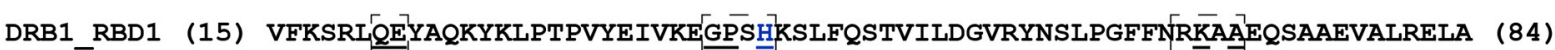
DRB2_RBD1 (1) MYKNQLQELAQRSCFNLPSYTCIREGPDHAPRFKATVNFNGEIFESPQYCSTLRQAEHSAAEVALNALS (70)

DRB1_RBD2 (101) KNLLQEYAQKMNYAIPLYQCQKVETLGRVTQFTCTVEIGGIKYTGAATRTKKDĀEISAGRTALIAIQS (170) DRB2_RBD2 (87) KNLLQEIAQRVGAPLPRYTTFR-SGLGHQPVFTGTVELAGITFTGDPAKNKKQAEKNAAMAAWSSLKQ (69)

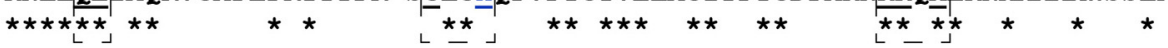
Region 1 Region 2 Region 3

FIGURE 6 | dsRNA binding domains of Arabidopsis DRB proteins. (A) Alignment of dsRBD structures of DRB1 (3ADG and 3ADJ) and predicted structure of DRB2, DRB3, DRB4, and DRB5 RBDs. Region 2 of non-canonical DRB1 dsRBD2 is shown (blue ribbon). (B) Amino acid sequence alignment of Arabidopsis DRB1 and DRB2. (C) Alignment of dsRNA binding domains of DRB1 and DRB2. Secondary folding structure is shown (top). dsRBD regions 1, 2, and 3 are boxed, and their conserved amino acids are underlined. The conserved histidine $(H)$ of region 2 is shown in blue, and arginine (R) in red. 

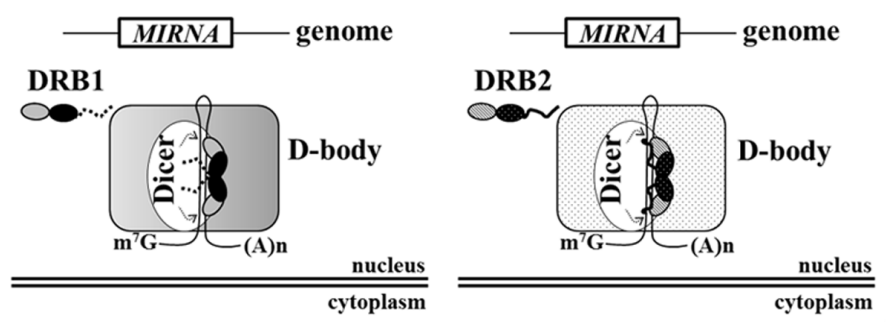

FIGURE 7 | Hypothetical model for the role of DRB1 and DRB2 domains. miRNAs are encoded as gene-like structures and are recognized by a dicer (DCL1) and a DRB protein. DRB1 dsRBD1 recognizes dsRNAs and its dsRBD2 promotes dimerization and interaction with other proteins, without the aid of its C-terminus (shown as a dotted line). DRB2 dsRBD1 also recognizes dsRNAs and its dsRBD2 appears to mediate different protein-protein interactions to those of DRB1, possibly assembling dicing bodies (D-bodies) different to those containing DRB1. This may also require the presence of the DRB2 C-terminus (shown as a continuous line).

studied their evolutionary conservation among land plants. The Arabidopsis DRB1 sequence was aligned to those of orthologs identified in public databases. The DRB1 orthologs were from a wide range of evolutionarily diverse plant species, including eudicots, monocots, and a moss. The alignment clearly showed a high degree of conservation in the N-terminal region relative to the whole sequence (Figure 4). Previously it has been shown that the severe developmental phenotype of Arabidopsis drb1 plants can be complemented via the transgene-based expression of a truncated version of Arabidopsis DRB1 (419 aa) lacking the 249 amino acid residues of the C-terminal domain (Wu et al., 2007; Yang et al., 2010). This is in accordance with our amino acid alignment because of the lack of C-terminal conservation, suggesting that the DRB1 C-terminus may not be required in all plant species. A lack of sequence conservation is also evident in region 2 of the second dsRBD of DRB1, dsRBD2. In canonical dsRNA binding domains, region 2 is characterized by a $\mathrm{GPxH}$ motif (where $\mathrm{x}$ is any residue), which is crucial for recognition of, and binding to, molecules of dsRNA (Gleghorn and Maquat, 2014). The dsRBD2 of Arabidopsis DRB1 has been shown to be a weak, non-canonically structured binding domain that primarily mediates protein-protein interaction, and not dsRNA recognition or binding (Yang et al., 2010, 2014). The lack of sequence conservation within region 2 may therefore account for the function of dsRBD2 in DRB1 in the miRNA pathway among land plants. The sequence conservation of DRB2 orthologs in land plants revealed two surprising findings: i) both DRB2 dsRBDs have very high sequence conservation, and ii) compared to the C-terminal region of plant DRB1 orthologs, the C-terminal region is markedly conserved (Figure 5). Taken together, these results suggest that DRB1 dsRBD2 has a conserved low affinity for dsRNA and that DRB2 has been more conserved in evolution than DRB1.

\section{Arabidopsis DRB dsRNA Binding Domains}

To study whether the lack of canonical structure of DRB1 dsRBD1 is unique to DRB1, the secondary structures for both
dsRBDs encoded by Arabidopsis DRB2, DRB3, DRB4, and DRB5 were predicted and superimposed onto the crystal structures of these domains of DRB1. This in silico analysis showed that dsRBD1 and dsRBD2 of DRB2, DRB3, DRB4, and DRB5 appear to adopt the canonical structure of DRB1 dsRBD1 (Figure 6A). The domain superimposition also revealed that region 2 , the loop between $\beta 1$ and $\beta 2$, was only re-oriented in DRB1 dsRBD2. This structural alteration to dsRBD2 has been previously suggested to reduce the dsRNA binding affinity of DRB1 (Yang et al., 2010). We then analyzed the amino acid sequence of DRB1 and DRB2 in more detail to gain insights into the secondary structures of these proteins. Although both DRB proteins are required in the miRNA pathway, their amino acid sequences are strikingly different, suggesting that they display different surfaces for protein-RNA and protein-protein interactions (Figure 6B).

In a canonical dsRNA binding domain, regions 1 and 2 bind dsRNA minor grooves while region 3 binds the dsRNA major groove (Gleghorn and Maquat, 2014). Region 1 is conserved in both dsRBDs of DRB1 and DRB2 (Figure 6C). Region 3 of DRB1 dsRBD2 and DRB2 dsRBD2 is also tightly conserved, but is dissimilar in their first dsRNA binding domain. Region 2 , in contrast, has the conserved GPxH motif in the dsRBD1 domains of both DRB proteins, but was only present in the dsRBD2 of DRB2. This region aids dsRNA binding to the major groove via the histidine $(\mathrm{H})$ residue of the $\mathrm{GPxH}$ motif, which is absent only in DRB1 dsRBD2 (Figure 6C). Nevertheless, when this residue was mutated in DRB1 dsRBD1, a dsRBD with high affinity to its dsRNA substrate, only a slight decrease in dsRNA binding affinity was observed (Yang et al., 2010). Taken together, these analyses provide evidence that DRB1 and DRB2 differ substantially at the amino acid and secondary structure levels.

\section{CONCLUSION}

Although our knowledge of the biogenesis of plant miRNAs has improved dramatically in recent years, several of the latest findings indicate that some important mechanisms remain poorly understood. The biogenesis of miRNA/miRNA* 
from miRNA-containing intermediates occurs in dicing bodies (D-bodies), and a growing number of genes, in addition to well characterized core components (e.g., DCL1, SE, and DRB1), have been shown to be required in this process (reviewed by Rogers and Chen, 2013). Thus, it is likely that the D-bodies are dynamic and may vary in protein composition according to developmental stage, environmental conditions and even precursor transcript structure. Although DRB1 is a well-characterized DCL1 partnering protein, our results reveal that DRB2 has been much more conserved during plant evolution. In addition, DRB1 and DRB2 have similar but functionally different domains, such as their dsRBD2 and C-terminus. The results presented here, together with our previous report (Reis et al., 2015), suggest that DRB1 and DRB2 act as bridging proteins in the assembly of different component proteins, and even different RNAs, into the core of the D-bodies, thus altering the properties of the D-bodies

\section{REFERENCES}

Altschul, S. F., Gish, W., Miller, W., Myers, E. W., and Lipman, D. J. (1990). Basic local alignment search tool. J. Mol. Biol. 215, 403-410. doi: 10.1016/S00222836(05)80360-2

Chang, K.-Y., and Ramos, A. (2005). The double-stranded RNA-binding motif, a versatile macromolecular docking platform. FEBS J. 272, 2109-2117. doi: 10.1111/j.1742-4658.2005.04652.x

Chen, C., Ridzon, D. A., Broomer, A. J., Zhou, Z., Lee, D. H., Nguyen, J. T., et al. (2005). Real-time quantification of microRNAs by stem-loop RT-PCR. Nucleic Acids Res. 33, e179. doi: 10.1093/nar/ gni178

Chendrimada, T. P., Gregory, R. I., Kumaraswamy, E., Norman, J., Cooch, N., Nishikura, K., et al. (2005). TRBP recruits the Dicer complex to Ago2 for microRNA processing and gene silencing. Nature 436, 740-744. doi: 10.1038 /nature 03868

Clarke, J. T., Warnock, R. C. M., and Donoghue, P. C. J. (2011). Establishing a time-scale for plant evolution. New Phytol. 192, 266-301. doi: 10.1111/j.14698137.2011.03794.x

Clough, S. J., and Bent, A. F. (1998). Floral dip: a simplified method for Agrobacterium-mediated transformation of Arabidopsis thaliana. Plant J. 16, 735-743. doi: 10.1046/j.1365-313x.1998. 00343.x

Coutu, C., Brandle, J., Brown, D., and Brown, K. (2007). pORE: a modular binary vector series suited for both monocot and dicot plant transformation. Transgenic Res. 16, 771-781. doi: 10.1007/s11248-0079066-2

Curtin, S. J., Watson, J. M., Smith, N. A., Eamens, A. L., Blanchard, C. L., and Waterhouse, P. M. (2008). The roles of plant dsRNA-binding proteins in RNAi-like pathways. FEBS Lett. 582, 2753-2760. doi: 10.1016/j.febslet.2008. 07.004

Dong, Z., Han, M.-H., and Fedoroff, N. (2008). The RNA-binding proteins HYL1 and SE promote accurate in vitro processing of pri-miRNA by DCL1. Proc. Natl. Acad. Sci. U.S.A. 105, 9970-9975. doi: 10.1073/pnas.0803 356105

Eamens, A. L., Kim, K. W., Curtin, S. J., and Waterhouse, P. M. (2012). DRB2 is required for microRNA biogenesis in Arabidopsis thaliana. PLoS ONE 7:e35933. doi: 10.1371/journal.pone.0035933

Eamens, A. L., Smith, N. A., Curtin, S. J., Wang, M.-B., and Waterhouse, P. M. (2009). The Arabidopsis thaliana double-stranded RNA binding protein DRB1 directs guide strand selection from microRNA duplexes. RNA 15, 2219-2235. doi: 10.1261/rna.1646909

Gleghorn, M., and Maquat, L. (2014). "Black sheep" that don't leave the doublestranded RNA-binding domain fold. Trends Biochem. Sci. 39, 328-340. doi: 10.1016/j.tibs.2014.05.003 and the functionality of the miRNA pathway as a whole (Figure 7).

\section{ACKNOWLEDGMENTS}

We thank the Australian Research Council (grant numbers DP130103697 and FF0776510) and contributions from the University of Sydney, Commonwealth Scientific and Industrial Research Organisation (CSIRO), Queensland University of Technology, and Australian Postgraduate Award scheme.

\section{SUPPLEMENTARY MATERIAL}

The Supplementary Material for this article can be found online at: http://journal.frontiersin.org/article/10.3389/fpls.2015.01201

Goodstein, D. M., Shu, S., Howson, R., Neupane, R., Hayes, R. D., Fazo, J., et al. (2012). Phytozome: a comparative platform for green plant genomics. Nucleic Acids Res. 40, D1178-D1186. doi: 10.1093/nar/gkr944

Haase, A. D., Jaskiewicz, L., Zhang, H., Lainé, S., Sack, R., Gatignol, A., et al. (2005). TRBP, a regulator of cellular PKR and HIV-1 virus expression, interacts with Dicer and functions in RNA silencing. EMBO Rep. 6, 961-967. doi: 10.1038/sj.embor.7400509

Han, M.-H., Goud, S., Song, L., and Fedoroff, N. (2004). The Arabidopsis double-stranded RNA-binding protein HYL1 plays a role in microRNAmediated gene regulation. Proc. Natl. Acad. Sci. U.S.A. 101, 1093-1098. doi: 10.1073/pnas.0307969100

Kurihara, Y., Takashi, Y., and Watanabe, Y. (2006). The interaction between DCL1 and HYL1 is important for efficient and precise processing of primiRNA in plant microRNA biogenesis. RNA 12, 206-212. doi: 10.1261/rna. 2146906

Larkin, M. A., Blackshields, G., Brown, N. P., Chenna, R., McGettigan, P. A., McWilliam, H., et al. (2007). Clustal W and Clustal X version 2.0. Bioinformatics 23, 2947-2948. doi: 10.1093/bioinformatics/btm404

Lee, H. Y., Zhou, K., Smith, A. M., Noland, C. L., and Doudna, J. A. (2013). Differential roles of human Dicer-binding proteins TRBP and PACT in small RNA processing. Nucleic Acids Res. 41, 6568-6576. doi: 10.1093/nar/ gkt361

Lee, Y., Hur, I., Park, S.-Y., Kim, Y.-K., Suh, M. R., and Kim, V. N. (2006). The role of PACT in the RNA silencing pathway. EMBO J. 25, 522-532. doi: $10.1038 /$ sj.emboj.7600942

Lu, C., and Fedoroff, N. (2000). A mutation in the Arabidopsis HYL1 gene encoding a dsRNA binding protein affects responses to abscisic acid, auxin, and cytokinin. Plant Cell 12, 2351-2365. doi: 10.1105/tpc.12.12.2351

Parker, G. S., Maity, T. S., and Bass, B. L. (2008). dsRNA binding properties of RDE4 and TRBP reflect their distinct roles in RNAi. J. Mol. Biol. 384, 967-979. doi: 10.1016/j.jmb.2008.10.002

Reis, R. S., Hart-Smith, G., Eamens, A. L., Wilkins, M. R., and Waterhouse, P. M. (2015). Gene regulation by translational inhibition is determined by Dicer partnering proteins. Nat. Plants 1, 14027. doi: 10.1038/nplants. 2014.27

Rogers, K., and Chen, X. (2013). Biogenesis, turnover, and mode of action of plant microRNAs. Plant Cell 25, 2383-2399. doi: 10.1105/tpc.113. 113159

Roy, A., Kucukural, A., and Zhang, Y. (2010). I-TASSER: a unified platform for automated protein structure and function prediction. Nat. Protoc. 5, 725-738. doi: $10.1038 /$ nprot.2010.5

Van Leene, J., Stals, H., Eeckhout, D., Persiau, G., Van De Slijke, E., Van Isterdael, G., et al. (2007). A tandem affinity purification-based technology platform to study the cell cycle interactome in Arabidopsis thaliana. Mol. Cell. Proteomics 6, 1226-1238. doi: 10.1074/mcp.M700078-MCP200 
Wu, F., Yu, L., Cao, W., Mao, Y., Liu, Z., and He, Y. (2007). The N-terminal doublestranded RNA binding domains of Arabidopsis HYPONASTIC LEAVES1 are sufficient for pre-microRNA processing. Plant Cell 19, 914-925. doi: $10.1105 /$ tpc. 106.048637

Yang, S. W., Chen, H.-Y., Yang, J., Machida, S., Chua, N.-H., and Yuan, Y. A. (2010). Structure of Arabidopsis HYPONASTIC LEAVES1 and its molecular implications for miRNA processing. Structure 18, 594-605. doi: 10.1016/j.str.2010.02.006

Yang, X., Ren, W., Zhao, Q., Zhang, P., Wu, F., and He, Y. (2014). Homodimerization of HYL1 ensures the correct selection of cleavage sites in primary miRNA. Nucleic Acids Res. 42, 12224-12236. doi: 10.1093/nar/gku907
Conflict of Interest Statement: The authors declare that the research was conducted in the absence of any commercial or financial relationships that could be construed as a potential conflict of interest.

Copyright $\odot 2016$ Reis, Eamens, Roberts and Waterhouse. This is an open-access article distributed under the terms of the Creative Commons Attribution License (CC BY). The use, distribution or reproduction in other forums is permitted, provided the original author(s) or licensor are credited and that the original publication in this journal is cited, in accordance with accepted academic practice. No use, distribution or reproduction is permitted which does not comply with these terms. 